1.

- \textit \{\} 


\title{
The Importance of HSPA-2 Expression in Laryngeal Squamous Cell Carcinomas
}

\author{
Onur Ceylan ${ }^{1}$ and Remzi Arslan ${ }^{2}$ \\ ${ }^{1}$ Affiliation not available \\ ${ }^{2}$ Ataturk University Faculty of Medicine
}

January 19, 2021

\begin{abstract}
Objective: Laryngeal Squamous Cell Carcinoma (LSCC) has high recurrence, metastasis and mortality rates, thus determining the markers related to prognosis and the methods that can be effective in its treatment are important. The number of studies on the prognostic role of HSPA in LSCC is very few. We did not find any study investigating the prognostic role of HSPA-2 in LSCC in the literature. The aim of our study was to examine the relationship between HSPA-2 expression and clinicopathological parameters in LSCC.

Material and Methods: HSPA-2 was studied on 104 cases who have been diagnosed with LSCC in the last 7 years. The connection between HSPA-2 expression and important prognostic parameters was investigated.

Results: In all of the cases, positive immunoreactivity was determined with HSPA-2. A significant relationship was determined between the HSPA-2 expression and important prognostic parameters such as lymphovascular invasion, lymph nodule metastasis, the primary tumor, the TNM stage, recurrence, metastasis and survival rates.

Conclusion: Our study supports the link between high HSPA-2 expression levels and poor prognostic parameters. Therefore we think that HSPA-2 can be used as a prognostic marker in laryngeal cancer. Also, we think that HSPA-2 is important in early diagnosis due to its increased expression even in LSCC precursor conditions.
\end{abstract}

Keywords: Laryngeal Squamous Cell Carcinoma; HSPA-2; Prognostic Factors

\section{Introduction}

While laryngeal cancer makes up 30-40\% of head and neck malignancies and 1-2.5\% of all malign cancers [1]. Every year, head and neck cancers are seen in more than half a million people worldwide, and one-fourth of these cases consists of laryngeal squamous cell carcinomas (LSCC) [2]. According to the GLOBOCAN 2018 data, the incidence and mortality rates of laryngeal cancer (100.000 per person, standardized rates according to age) are 3.6/100.000 and 1.9/100.000 in males and; 0.5/100.000 and 0.3/100.000 in females respectively [3]. It has been reported that laryngeal cancer is seen 7 times more frequently in males and it makes up $1 \%$ of deaths related to malignancies in the population and $1.6 \%$ in males [4]. Laryngeal carcinomas are $95-$ $98 \%$ squamous cell carcinomas (SCC), and it takes the second place after lung cancer in respiratory system malignancies [2].

LSCC has high recurrence, metastasis and mortality rates [2]. Approximately $60 \%$ of patients are advanced (Stage III and IV) at the time of diagnosis [5]. Although the incidence of laryngeal cancer has declined, the 5-year survival rate has dropped from $66 \%$ to $63 \%$ in the last 40 years [6]. When all these factors are 
considered, understanding the pathogenesis of this cancer, determining its prognostic parameters and the markers related to these parameters are quite important.

Heat shock proteins, a large chaperon protein group (Heatshock-related 70-kDa protein) (HSP), are divided into a few different subgroups according to some of their characteristics [7]. They have a protective function for the cell by creating HSP adaptation mechanisms and it makes an important step in the defense system [8]. HSPA (HSP70), make up the largest group of HSP's and the genes for this family display many different functions, localization and expression [7]. HSPA-2, also known as HSP70-2 in HSPA family is a significant member that has not been studied yet. It has been defined as a protein specific to the testicles having a fundamental role in primary spermatocytes and spermatogenesis in spermatids for the first time [9]. In addition, it has been reported that HSPA-2 can be seen in non-testicular tissues and has an important role especially in carcinogenesis, and is necessary for tumor growth [10].

In the literature, there are various studies on this topic in different organs [11-17]. The number of studies on the prognostic role of HSPA in LSCC is very few [8]. Besides, we did not find any study investigating the prognostic role of HSPA-2 in LSCC in the literature. In our study, the aim was to examine the relationship between HSPA-2 expression and clinicopathological prognostic parameters in LSCC.

\section{Material and Method}

\section{Patients' General Information and Tissue Specimens}

In our study, laryngectomy materials examined between January 2009 and December 2015 were investigated retrospectively. 114 cases who were diagnosed with LSCC and have not previously received chemotherapy and radiotherapy were included in the study. Previous chemotherapy and/or radiotherapy, 8 patients were excluded. The clinical information of the patients was obtained from the automation system of our hospital. Recurrence and metastasis were determined by imaging methods, clinical examination epicrisis and examination of pathological materials sent to our department in the postoperative period. In order to determine the survival rate, recurrence and metastasis, the follow-up period for all 114 patients was determined as 5 years, and changes after 5 years were not taken into account. Pathology reports, slides and paraffin blocks of the patients were extracted from our archive. Paraffin blocks containing adjacent non-cancerous tissues of at least $1 \mathrm{~cm}$ in length were selected for the immunohistochemical study.

\section{Immunohistochemistry}

4-micron sections were taken from the blocks where the tumor was the densest and were put into the Roche Ventana automatic immunohistochemistry staining device (Ventana Roche, ABD) after the tissues placed on charged slides in a 70-degree drying oven for 15 minutes. The tissues in the device were treated with ULTRA Cell Conditioning Solution, hydrogen peroxide, HSPA-2 antibodies (Nova Castra, Leica, Newcastle, United Kingdom) after being subjected to deparaffinization and dehydration processes respectively.

The immunohistochemical stains were evaluated by two pathologists. As negative control group adjacent squamous epithelium not containing dysplasia were accepted. Nuclear and/or cytoplasmic staining was considered positive for HSPA-2. For HSPA-2, staining rate=0 (Grade-0), without staining $1-10 \%=1$ (Grade1), $11-49 \%=2$ (Grade-2), [?]50\% = 3 (Grade-3) were accepted. HSPA-2 was evaluated as follows; staining intensity: 0; no staining (Grade-0), 1+; weak cytoplasmic and nuclear staining (Grade-1), 2+; moderate cytoplasmic and nuclear staining (Grade-2), 3+; strong cytoplasmic and nuclear staining (Grade-3) (Figure 1). The immunoreactivity score (histoscore) was calculated using the method of multiplying the intensity and rate. The histoscore values for HSPA-2 0 were evaluated as follows; negative (Grade-0), 1-3; weak (Grade-1), 4-6; moderate (Grade-2), 7-9 strong (Grade-3) [15]. The connection between HSPA-2 staining rate, intensity, histoscore and age, gender and important prognostic parameters such as histological grade, the TNM stage, the primary tumor, lymph nodule metastasis, the presence of lymphovascular invasion, survival rate, recurrence and metastasis was examined.

Our study was approved by our local ethics committee (08-31/26.12.2019). 


\section{Results}

\section{Patients' Demographic and Histopathological Features}

The mean age of the 104 total cases in our study was determined as 56 \pm 8.1 years (33-82 years). There was no significant difference between the mean age of both genders (p: 0.775). 102 of the cases were male, and the male/female ratio was 8,5/1. Glottic cancers $(75.4 \%)$ were more common than supraglottic cancers $(22 \%)$, and three primary subglottic cancers were recorded $(2.6 \%)$. The average macroscopic radius of the tumor was determined as $3.1 \pm 1.8(0.8-6 \mathrm{~cm}) \mathrm{cm}$. In the histological grading of the cases, it was determined that 70 of the cases were moderately differentiated (G2). Primary Tumor (pT) distributions were observed as follows: 12 of them had pT1, 18 of them had pT2, 36 of them had pT3 and 48 of them had pT4. According to the TNM staging system (American Joint Committee on Cancer) [18], 12 cases were stage I, 16 cases were stage II, 26 cases were stage III, 60 cases were stage IV. Lymphovascular invasion was present in 66 cases but was not observed in 48 of the cases. When the peritumoral lymphocytic reaction distribution was examined, it was determined that 52 of the cases were of moderate intensity. When the lymph nodule metastasis distributions of the cases were examined, it was determined that 78 cases did not have lymph nodule metastasis, and 28 of them had pN1. $60(52,6 \%)$ of the patients died within 5 years of observation. The survival rate ranged from 1 months to 52 months. Metastasis was seen in $24(21 \%)$ patients, recurrence was seen in $8(7 \%)$ patients within 5 years of observation. (Table 1 ).

\section{Expression of HSPA-2 in different areas of laryngeal tisues}

A total of 114 cases of paraffin blocks of LSCC and adjacent non-cancerous tissues were analyzed by immunohistochemistry. In all of the cases, HSPA-2 positivity was determined in tumoral areas (\%100). The positive expression rate of HSPA-2 in the adjacent non-cancerous tissues was 48/114 (\%42). There was significant correlation between adjacent non-cancerous tissues and tumoral areas (p:0,002). There was no significant correlation between adjacent non-cancerous tissues and normal tissues (p: 0,225).

In well-differentiated tumors, a more distinct expression was observed predominately in the periphery of tumor solid islands. In areas where differentiation loss was pronounced, more widespread and severe expression was observed. Additionally, while positivity was observed only in $1 / 3$ of the basal part of the epithelium in low-grade epithelial dysplasia areas, full coat staining was seen in high-grade epithelial dysplasia areas (Figure 2).

\section{Prognostic significance of HSPA-2 expression in LSCC}

The statistical relationship of age and the HSPA-2 staining ratio, intensity, histoscore could not be determined (p: 0,527/p: 0,722/p: 0,518). The statistical relationship of gender and the HSPA-2 staining ratio, intensity, histoscore could not be determined either (p: 0,235/p: 0,255/ p: 0,092).

When the interactions between prognostic parameters such as the tumoral macroscopic volume, peritumoral lymphocytic reaction, the presence of ulceration, perineural invasion, , lymphovascular invasion, histological grade, lymph nodule metastasis, primary tumor, the TNM stage, recurrence, metastasis and survival rate were examined, the following results were obtained. A significant positive correlation was observed between the presence of lymph nodule metastasis $(\mathrm{pN})(\mathrm{p}: 0,0046, \mathrm{p}: 0,0008, \mathrm{p}: 0,0014)$, lymphovascular invasion (p: 0,0003, $\mathrm{p}: 0,0009, \mathrm{p}: 0,0001)$, primary tumor $(\mathrm{pT})(\mathrm{p}<0,0001, \mathrm{p}<0,0001 \mathrm{p}<0,0001)$, TNM stage $(\mathrm{p}<0,0001, \mathrm{p}<0,0001$ $\mathrm{p}<0,0001)$, recurrence (p:0,0020, p:0,0010, p:0,0009), metastasis (pM) $(\mathrm{p}<0,0001, \mathrm{p}<0,0001 \mathrm{p}<0,0001)$, survival rate and HSPA-2 staining rate, staining intensity, histoscore (Table-2). A significant positive correlation was observed between the tumoral macroscopic volume (p: 0,015, p:0,014) and HSPA-2 staining rate and histoscore only. A statistically significant relationship was not found between histological grade (p:0,6896, p:0,2405, p:0,2434), peritumoral lymphocytic reaction (p:0,0975, p:0,2431, p:0,5526), ulceration (p:0,3696, p:0,4774, p:0,2143), perineural invasion (p:0,1543, p:0,1291, p:0,054) and HSPA-2 staining rate, intensity and histoscore (Table-2).

Laryngeal cancer is most frequently seen between the 5. and 7. decades of life in the world [1]. Laryngeal cancers include $1.1 \%$ of all malignancies worldwide and this rate is $1.9 \%$ in males, and $0.3 \%$ in females [4]. 
In our study similar to literature, the mean age of the patients was $56 \pm 8.1$ and male/female ratio was 8 .

Heat shock proteins are chaperon proteins that have a protective function for the cell by normally creating an adaptation mechanism and increasing against environmental and pathophysiological stressors, and they make up an important step in the defense system. HSP70t and HSP70-2 (HSPA-2), unlike other HSP70 chaperon proteins, are expressed in the testicular tissues and are not seen in other tissues or are rarely seen in them [19]. In a study conducted on rats, Zhu et. al. stated that when they caused HSPA-2 defects, the primer spermatocytes got stuck at meiosis I, and other HSPA proteins cannot compensate for it. According to these results, they have argued that HSPA-2 is a molecular chaperone for CDC2/cyclin B1 complex formation, and that their interaction is necessary to ensure the G2/M-phase transition during mitosis and meiotic cell cycles [20]. In a different study, it has been noted that the G1/S phase arrest occurred, and malign cell proliferation decreased after the HSPA-2 gene was suppressed in the lung adenocarcinomas. It was shown that after the HSPA-2 decreased; the ERK1/2 cascade having functions such as cell survival rate, proliferation stimulation, cell differentiation, cell aging and apoptosis was affected [21].

It has been reported that HSPA-2 is effective in many malignancy carcinogenesis. While it is not expressed in normal tissues, it is excessively expressed in malign cells [14]. The excessive expression of HSPA-2 has been shown in malignancies in the pancreas [11], cervix [12], bladder [13], lungs [14], esophagus [15], nasopharynx [16] and liver [17]. All of these studies show that HSPA-2 is effective in the formation and development of tumors. In our study we have confirmed that HSPA-2 is expressed in LSCC. In all of the cases, HSPA-2 positivity was determined in tumoral areas (\%100). The positive expression rate of HSPA-2 in the adjacent non-cancerous tissues was 48/114 (\%42). There was significant correlation between adjacent non-cancerous tissues and tumoral areas. HSPA-2 expression was only observed in the basal layer of adjacent squamous epithelium not containing dysplasia in all of the cases. In well-differentiated tumors expression was observed predominantly in the periphery of tumor solid islands, and a more intense expression was observed in a more diffused way in areas where differentiation loss was distinct in tumors cells. A diffused and strong expression being observed in moderately and poorly differentiated tumors while weak staining seen in the adjacent epithelial basal and the periphery of well-differentiated tumor islands have suggested that HSPA-2 might have a role in tumor cell migration, invasion and carcinogenesis.

Zhang et. al. reported that while they did not observe staining with HSPA-2 in the normal esophagus epithelial, they observed poor staining in areas adjacent the tumor and squamous epithelium basal layer, and stronger staining in infiltrative areas [15]. In their study, Screglnska et. al. reported observing positive HSPA-2 in keratinocytes in the adjacent epidermis basal layer and in the infiltrative areas of all the skin tumors. On the other hand, they reported that they observed positivity with HSPA-2 in infiltrative areas in half of their cases, while no positivity was observed in the normal epithelium in the areas adjacent to the tumor in SCC in the head and neck region [14].

A different point that in our study we have observed important differences with staining rate and intensity in the low-grade epithelial dysplasia and high-grade epithelial dysplasia areas of the adjacent epithelium. In all of our cases in low-grade epithelial dysplasia areas, positivity was found in only $1 / 3$ of the basal part of the epithelium, while a full coat strong staining was seen in high-grade epithelial dysplasia areas. With this different staining pattern, we think that the HSPA-2 expression pattern can be extremely helpful in differential diagnosis when distinguishing epithelial dysplasia in laryngeal biopsies. No study has been conducted in the literature on this subject, and we think that new studies should be conducted with a larger number of cases.

In addition to HSPA-2 being excessively expressed in many types of cancer, there are many studies showing that it has an effect on tumor progression. It is indicated that tumor cells gain resistance against apoptosis related to hypoxia with an increase of HSPA-2, and that is how they stay alive [22]. In our study, a significant relationship was observed between the excessive expression of HSPA-2 and important prognostic parameters such as lymphovascular invasion, the primary tumor (pT), lymph nodule metastasis ( $\mathrm{pN})$, metastasis (pM), recurrence, the TNM stage and survival rate. Lymphatic metastasis is one of the most important reasons for poor prognosis and therefore low survival rate in carcinomas. The strong relationship between lymphovas- 
cular invasion and HSPA-2 expression in our study emphasized the prognostic importance of HSPA-2. Also lymph node metastasis $(\mathrm{pN})$, primary tumor $(\mathrm{pT})$, metastasis $(\mathrm{pM})$ are the most important determinants in terms of staging and prognosis. In our study, a significant relationship was observed between all three and HSPA-2 expression. One of the most important points in our study is the link between the survival of patients and HSPA-2 expression. With the Kaplan Meier analysis of overall survival method, we found that patients with increased HSPA-2 expression at all stages had shorter survival regardless of other prognostic parameters (Table 3). It is known that HSPA-2 migrates to the nucleus and nucleoli under stress conditions where it is localized in the cytoplasm under normal conditions. This is critical for cell migration, tumor formation and metastasis, as HSPA2 moves continuously between the cytoplasm and the nucleus in a heat shock state [23]. In our study, staining in cytoplasm and nucleus with HSPA-2 was observed in the infiltrative areas. We also observed stronger nuclear staining in patients with metastases compared to those not seen.

$\mathrm{Fu}$ et. al. reported that histological grade, tumor radius, clinical stage in hepatocellular carcinomas were directly proportionate to HSPA-2, thus HSPA-2 can have a role in carcinogenesis, tumor development, progression and metastasis. They indicated that they found a significant negative correlation especially between survival rate and HSPA-2 expression [17]. In their study emphasizing on esophagus SCC cases, Zhang et. al. stated that the excessive expression of HSPA-2 was associated with the primary tumor, clinical stage, lymph nodule metastasis and recurrence [15]. Garg et. al. have argued that HSPA-2 expression is related to cellular motility and growth, so HSPA-2 has a role in cancer spread during the early stages. In their study on bladder cancer, they stated that they observed a significant relationship between the clinical stage, histological grade and HSPA-2 expression [13]. In a different study conducted by Garg et. al. on cervix cancer, they obtained similar results and indicated that HSPA-2 had an effect on the progression of cervix cancer [12]. In their study, Zhai et. al. have noted that HSPA-2 is related to a poor prognosis in tumor angiogenesis and pancreatic adenocarcinoma, and has an important place in its progression [11]. Similar to our study, various studies have shown that in cases where excessive expression of HSPA-2 is seen, there are higher lymph nodule metastasis and therefore a worse prognosis [13, 24]. In their study, Scieglınska et. al. found a significant relationship between HSPA-2 expression and the TNM stage, histological grade and prognosis in Non-Small Cell Lung Carcinomas. They have highlighted that especially in stage I and II cases, high HSPA-2 expression is related to lower survival rates [14]. In their in vivo study using a mouse model, Garg et. al. considered the matter from a different point of view and stated that they decreased tumor growth rates by $75 \%$ with HSPA-2 inhibition in rats [12]. When the results of our study and the available data in the literature were evaluated, finding a significant relationship between important prognostic parameters such as the primary tumor, lymphovascular invasion, lymph nodule metastasis, the TNM stage, metastasis, recurrence, survival rate suggested that this marker can be used in prognosis in LSCC by immunohistochemically. Especially in our study, it has been shown for the first time that HSPA-2 can be an important prognostic indicator in LSCC.

\section{Conclusion}

Our study supports that there is an association between high HSPA-2 expression levels and poor prognostic parameters. According to our results, we believe that HSPA-2 can be used as a prognostic marker in laryngeal cancer. There are different studies on this in the literature. However, there is a limited number of studies conducted with immunohistochemical method. In this respect, our study shows that HSPA-2 gives significant results with immunohistochemical methods as well. Also, we think that HSPA-2 is important in early diagnosis due to its increased expression even in LSCC precursor conditions.

\section{Acknowledgments}

No acknowledgments to declare

\section{Conflict of interest statement}

All authors declared that there is no conflict of interest.

\section{Financial Support}


None

\section{References}

\section{References}

1. Markou, K., et al., Laryngeal cancer: epidemiological data from Northern Greece and review of the literature. Hippokratia, 2013. 17(4): p. 313.

2. $\quad$ Fong, P.Y., et al., Association of clinical factors with survival outcomes in laryngeal squamous cell carcinoma (LSCC). PloS one, 2019. 14(11): p. e0224665.

3. Bray, F., et al., Global cancer statistics 2018: GLOBOCAN estimates of incidence and mortality worldwide for 36 cancers in 185 countries. CA: a cancer journal for clinicians, 2018. 68(6): p. 394-424.

4. $\quad$ Ferlay, J., et al., Cancer incidence and mortality worldwide: sources, methods and major patterns in GLOBOCAN 2012. International journal of cancer, 2015. 136(5): p. E359-E386.

5. Groome, P.A., et al., Management and outcome differences in supraglottic cancer between Ontario, Canada, and the Surveillance, Epidemiology, and End Results areas of the United States. Journal of clinical oncology, 2003. 21(3): p. 496-505.

6. Siegel, R.L., K.D. Miller, and A. Jemal, Cancer statistics, 2016. CA: a cancer journal for clinicians, 2016. 66(1): p. 7-30.

7. Scieglinska, D., et al., Differential expression of HSPA1 and HSPA2 proteins in human tissues; tissue microarray-based immunohistochemical study. Histochemistry and cell biology, 2011. 135(4): p. 337-350.

8. Aleksandra Barańska, A., et al., Expression of heat shock protein 70 in the tissue of patients with laryngeal squamous cell carcinoma. European Journal of Clinical and Experimental Medicine, 2019(1): p. $16-21$.

9. Dix, D.J., et al., HSP70-2 is required for desynapsis of synaptonemal complexes during meiotic prophase in juvenile and adult mouse spermatocytes. Development, 1997. 124(22): p. 4595-4603.

10. Rohde, M., et al., Members of the heat-shock protein 70 family promote cancer cell growth by distinct mechanisms. Genes \& development, 2005. 19(5): p. 570-582.

11. Zhai, L.-L., et al., Overexpressed HSPA2 correlates with tumor angiogenesis and unfavorable prognosis in pancreatic carcinoma. Pancreatology, 2017. 17(3): p. 457-463.

12. Garg, M., et al., Germ cell-specific heat shock protein 70-2 is expressed in cervical carcinoma and is involved in the growth, migration, and invasion of cervical cells. Cancer, 2010. 116(16): p. 3785-3796.

13. Garg, M., et al., Heat-shock protein 70-2 (HSP70-2) expression in bladder urothelial carcinoma is associated with tumour progression and promotes migration and invasion. European Journal of Cancer, 2010. 46(1): p. 207-215.

14. Scieglinska, D., et al., HSPA2 is expressed in human tumors and correlates with clinical features in non-small cell lung carcinoma patients. Anticancer research, 2014. 34(6): p. 2833-2840.

15. Zhang, H., et al., Overexpression of HSPA2 is correlated with poor prognosis in esophageal squamous cell carcinoma. World journal of surgical oncology, 2013. 11(1): p. 141

16. Jalbout, M., et al., Polymorphism of the stress protein HSP70-2 gene is associated with the susceptibility to the nasopharyngeal carcinoma. Cancer letters, 2003. 193(1): p. 75-81.

17. Fu, Y., et al., Expression of HSPA2 in human hepatocellular carcinoma and its clinical significance. Tumor Biology, 2014. 35(11): p. 11283-11287.

18. Amin, M.B. and S.B. Edge, AJCC cancer staging manual. 2017: springer. 
19. Fourie, A.M., P.A. Peterson, and Y. Yang, Characterization and regulation of the major histocompatibility complex-encoded proteins Hsp70-Hom and Hsp70-1/2. Cell stress \& chaperones, 2001. 6(3): p. 282.

20. Zhu, D., D.J. Dix, and E. Eddy, HSP70-2 is required for CDC2 kinase activity in meiosis I of mouse spermatocytes. Development, 1997. 124(15): p. 3007-3014.

21. Cao, L., et al., Downregulation of HSPA2 inhibits proliferation via ERK1/2 pathway and endoplasmic reticular stress in lung adenocarcinoma. Annals of Translational Medicine, 2019. 7(20).

22. Xia, L.-M., et al., Hypoxia induces heat shock protein HSP70-2 expression in a HIF-1 dependent manner. Zhonghua gan zang bing za zhi= Zhonghua ganzangbing zazhi= Chinese journal of hepatology, 2009. 17(3): p. 207-212.

23. Ścieglińska, D., et al., The HspA2 protein localizes in nucleoli and centrosomes of heat shocked cancer cells. Journal of cellular biochemistry, 2008. 104(6): p. 2193-2206.

24. Mestiri, S., et al., Genetic variation in the tumor necrosis factor- $\alpha$ promoter region and in the stress protein hsp70-2: Susceptibility and prognostic implications in breast carcinoma. Cancer, 2001. 91(4): p. $672-678$.

\section{Figure Legends}

\section{Figure-1}

A-1: Histopathological view of HSPA2 weak staining in infiltrative areas. A-2: Modarate staining. A-3: andA-4: Strong Staining.

\section{Figure-2 (HSPA-2 expression)}

B-1: In the basal layer of the adjacent epithelium and more distinct expression in the periphery of tumor solid islands.B-2: In the basal layer of the adjacent epithelium and full coat staining in high-grade epithelial dysplasia areas. B-3:Intense expression in areas with distinct differentiation loss in the tumor cells. B-4: Staining in the 1/3 of the basal part of the epithelium in low-grade epithelial dysplasia areas. B-5: Full coat staining in high-grade epithelial dysplasia areas. B-6:More distinct expression in the periphery of tumor solid islands. 